\title{
Investigation of an acute chemical incident: exposure to fluorinated hydrocarbons
}

Ronan A Lyons, Dorothy Wright, Hilary M P Fielder, Michael McCabe, Andar Gunneberg,Pam Nash, Phillip Routledge, Huw Rees

\begin{abstract}
Objectives-To assess whether attendance at the site after an incident in a sewer was associated with symptoms in emergency personnel and whether the prevalence of symptoms was associated with estimated levels of exposure to any chemical hazard. Methods-Symptoms experienced by people attending an incident involving two dead sewer workers suggested the presence of a chemical hazard, before environmental sampling confirmed any toxic agent. Self reported symptoms, estimated exposures, and biomarkers of exposure for likely agents from all 254 people who attended the incident and a referent occupational group matching the 83 emergency personnel who went to the Accident and Emergency department (A and $E$ ) in the first 48 hours were recorded. The prevalence of symptoms and concentrations of creatine phosphokinase in serum of the 83 early patients at $A$ and $E$ were compared with their referent occupational group. In all workers who attended the incident, the trends in symptom prevalences and concentrations of creatine phosphokinase in serum were examined by distance from the site and predefined exposure category.
\end{abstract}

Results-Among all workers who attended the incident, symptoms of shortness of breath and sore throat were significantly associated with indirect estimates of exposure but not associated with concentrations of creatine phosphokinase. Freon was detected in two blood samples. The early patients at $A$ and $E$ reported more symptoms than their matched reference group and their median concentrations of creatine phosphokinase were higher.

Conclusions-The association between symptoms and concentrations of creatine phosphokinase with attendance at the site indicated the presence of a continuing hazard at the site and led to extra precautions being taken. Comparison values from the referent occupational group prevented unnecessary medical follow up. (Occup Environ Med 2000;57:577-581)

Keywords: pollution; chemical; population

Accident and

Emergency Medicine,

Neath General

Hospital, Wales, UK

P Nash

Correspondence to:

Dr H Fielder

fielderhm@cardiff.ac.uk

Accepted 2 May 2000 workers are hypoxia caused by the accumulation of methane and carbon dioxide, hydrogen sulphide poisoning, and explosion, although occasional reports of unidentifed toxic gas have been reported. ${ }^{1}$ Rescue workers and accident and emergency (A and E) staff who examined the bodies complained of a strong smell of chemicals and experienced various symptoms including headache, drowsiness, shortness of breath, sore throat, and burning skin. Despite these findings, various responders doubted that this was a serious chemical incident until there was objective evidence from environmental sampling, and while these tests were being undertaken many emergency personnel, local authority and agency staff attended the scene of the incident. Over the next 2 days more than 80 symptomatic or concerned people attended an $\mathrm{A}$ and $\mathrm{E}$ department and 17 people were admitted to hospital. This paper describes a study of estimated exposures, serum biomarkers of exposure, and self reported symptoms in people who attended the site. It shows the importance of epidemiological study, especially when the chemical hazard is unknown, to assess the risk to emergency responders, to manage the acute incident, and to inform clinical follow up of patients at the A and E department.

The aim of the study was to find out whether attendance at the site was associated with symptoms and whether the prevalence of symptoms was associated with estimated concentrations of exposure.

\section{Method}

Initial histories of symptoms and potential exposures were taken from 83 emergency responders who attended the $\mathrm{A}$ and $\mathrm{E}$ department (10-13 October) and blood samples were taken between 10 and 17 October. A frequent finding among these early patients was an increased level of creatine phosphokinase (CK). There was some uncertainly about the correct upper limit of $\mathrm{CK}$ in a predominantly healthy young population as normative data used in hospital are based on presenting samples from a wider population. Therefore, baseline symptom data and blood samples were collected between 15 and 17 October on a referent group, to match the early patients at A and $\mathrm{E}$. The referent group was selected on the basis of occupational matching with an attempt to match equal numbers of police, ambulance, local authority, and environment agency staff, and chosen from the same base stations as the early patients. No attempt was made to match on smoking as this information was not collected from the exposed group.

Subsequently, all people who had been at the site of the incident between 10 and 14 October were asked to attend a special clinic between 21 
October and 8 November (most by 25 October) at the A and E Department for a check up. At this appointment a questionnaire was used to collect data on the presence or absence of 14 symptoms, date and time of onset, the presence of longstanding health problems or symptoms, and medication used. People were asked to mark the place, the date, and time that they were present at the incident on a high definition map and to indicate whether they had used protective equipment. Blood samples taken for urea and electrolytes, cardiac enzymes, and liver function tests were analyzed at the hospital laboratory, where the upper limit of normality for CK is set at 175 units. Gas chromatography-mass spectroscopy (GC-MS) of samples taken from the sewer effluent in the chamber on the day of the incident showed the presence of small quantities of $\alpha$-methyl styrene and large quantities of Freon11. Blood samples from people attending the hospital and the case referent group were sent to the Health and Safety Executive Laboratory at Sheffield for toxicological analysis of $\alpha$-methyl styrene, Freon, and volatile hydrocarbons. Headspace GC-MS was used to screen for volatiles followed by full scan analysis for Freon-11, $\alpha$-methyl styrene, styrene, and hexanal with selected ion monitoring GS-MS. Samples of urine were tested for atrolactic acid, a metabolic product of $\alpha$-methyl styrene; for fluoride, a possible product of Freon- $11^{2}$; and myoglobin for evidence of muscle damage.

Severity of exposure was estimated in two ways: with distance from the chamber as a proxy of exposure and by an exposure classification devised before any analysis, based on the period of exposure, the use of protective equipment (including breathing apparatus), and distance from the chamber measured in metres (nearest distance taken to be $1 \mathrm{~m}$ ). Thus, the exposure categories were defined as high (people sampling from the chamber without protective clothing or having been less than $5 \mathrm{~m}$ from the chamber on 10-12 October without protective clothing); medium (people 5-30 m on 10-15 October without protective clothing or less than $5 \mathrm{~m}$ on 13-15 October without protective clothing); low (those $31-49 \mathrm{~m}$ on 10-15 October without protective clothing), and very low exposure (those at $50 \mathrm{~m}$ or more on any day, or those who were nearer and wearing protective clothing).
Statistical analysis was carried out with SPSS for windows and Epi-Info. The $\chi^{2}$ test for trend was used to determine whether symptom prevalence in all workers who attended the incident was related to distance from the site or to exposure category. The prevalence of symptoms was compared between exposed and unexposed occupational groups with the $\chi^{2}$ test and Fisher's exact test as appropriate; the MannWhitney $U$ test was used to compare the distribution of CK concentrations between groups.

Information and samples were also collected from workers and residents in premises adjacent to the sewage chamber but these are not included in the analyses presented here, which focus on the hypothesised airborne exposure with the chamber as its source. Comments on their results are included in the discussion.

\section{Results}

Two hundred and fifty eight people were known to have attended the site and data were collected on 254. Lists of other employees not directly involved with the primary emergency response were not kept but it was thought that most of these subsequently attended $\mathrm{A}$ and $\mathrm{E}$ and are included in the cohort. Comparison between initial history as recorded at first A and $\mathrm{E}$ visit and subsequent history by questionnaire showed a high degree of concordance, with $91 \%$ agreement between symptoms recorded at the initial $A$ and $E$ visit and subsequent questionnaire.

Seventeen people were admitted to hospital for observation with severe symptoms. Most had chest pain or shortness of breath, some with raised concentrations of $\mathrm{CK}$ but not raised aspartate transaminase. One patient with severe respiratory symptoms was found to have widespread bronchial ulcerations on bronchoscopy. Another patient had a cardiac arrythmia on electrocardiogram.

Among the first $83 \mathrm{~A}$ and $\mathrm{E}$ patients, 25 were classified as having a high exposure at the site, 28 a medium exposure, 13 low exposure, and three very low exposure. Eight had exposures relating to handling the bodies and exposure data were missing for four people. Baseline comparison between the first $83 \mathrm{~A}$ and $\mathrm{E}$ patients and 83 in the referent group showed a good match: mean age 38.7 versus 39.8 years, $86 \%$ versus $75 \%$ men, current use of medication $12.3 \%$ versus $19.3 \%$, and longstanding

Table 1 Prevalence of symptoms in early patients at accident and emergency and the referent group

\begin{tabular}{lccll}
\hline Symptom & $\begin{array}{l}\text { Prevalence in early } \\
\text { patients } n(\%)\end{array}$ & $\begin{array}{l}\text { Prevalence in referent } \\
\text { group n (\%) }\end{array}$ & $\begin{array}{l}\text { Relative rate for early } \\
\text { patients }\end{array}$ & 95\% CI (p value) \\
\hline Abdominal pain & $12(14.8)$ & $4(4.8)$ & 3.07 & 1.03 to $9.04(0.04)$ \\
Chest pain & $8(9.9)$ & $0(0.0)$ & - & $(0.003)$ \\
Cough & $22(27.2)$ & $10(12.0)$ & 2.25 & 1.14 to $4.46(0.02)$ \\
Diarrhoea & $12(14.8)$ & $3(3.6)$ & 4.10 & 1.20 to $14.0(0.02)$ \\
Drowsiness & $15(18.5)$ & $4(4.8)$ & 3.89 & 1.33 to $11.1(0.007)$ \\
Headache & $42(51.9)$ & $17(20.5)$ & 2.53 & 1.58 to $4.06(<0.001)$ \\
Itchy eyes & $16(19.8)$ & $2(2.4)$ & 1.82 & 0.85 to $3.88(0.13)$ \\
Nausea & $10(12.3)$ & $4(4.8)$ & 4.12 & 1.16 to $22.7(0.02)$ \\
Shortness of breath & $19(23.5)$ & $4(4.8)$ & 4.87 & 1.73 to $13.7(<0.001)$ \\
Skin rash & $10(12.3)$ & $2(2.4)$ & 3.56 & 0.84 to $7.84(0.09)$ \\
Sore throat & $36(44.4)$ & $4(4.8)$ & 3.07 & 1.73 to $5.47(<0.001)$ \\
Vomiting & $7(8.6)$ & $4(4.8)$ & 5.59 & 0.77 to $16.8(0.17)$ \\
Weakness & $23(28.4)$ & 3.29 & 1.13 to $16.3(<0.001)$ \\
Wheeze & $13(16.0)$ & & & 1.12 to $9.67(0.02)$ \\
\hline
\end{tabular}




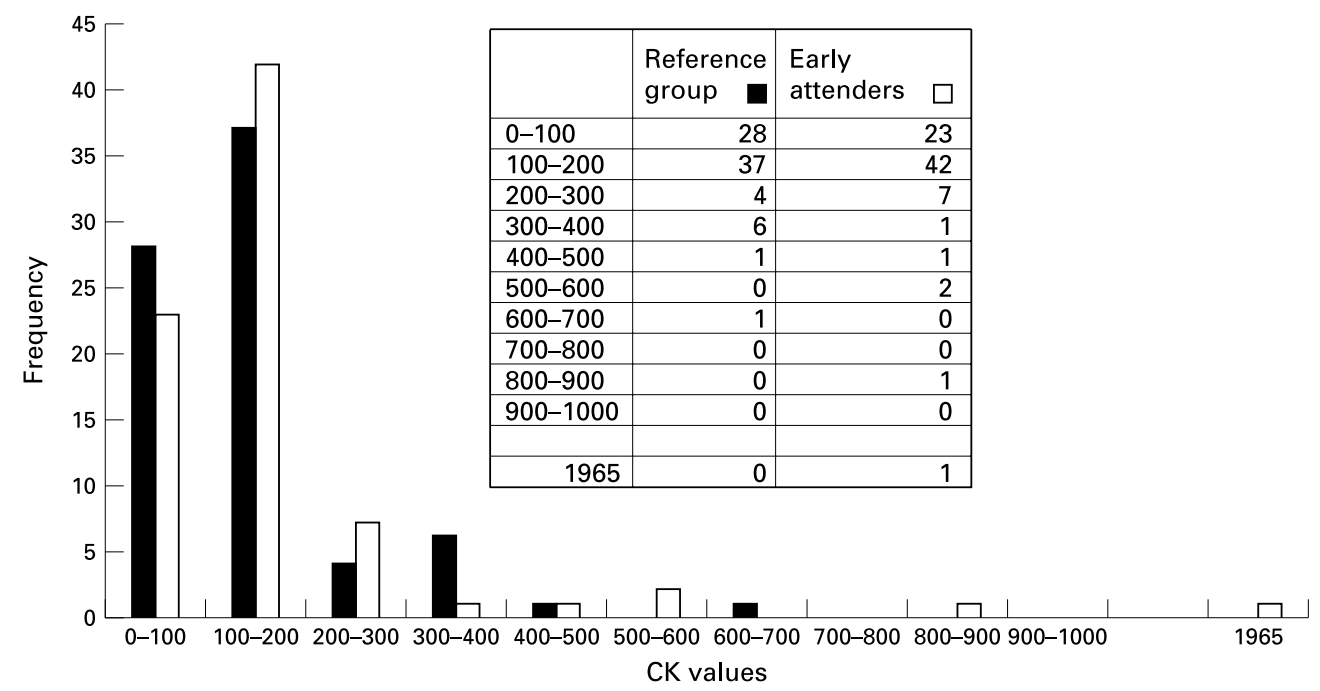

Frequency distributionof $C K$ for early attenders and the referent group.

illness $11.1 \%$ versus $15.7 \%$. Policemen accounted for $29 \%$ of the exposed group and $31 \%$ of the control group, local authority workers $24 \%$ versus $40 \%$, firemen $24 \%$ versus $12 \%$, ambulance personnel $5 \%$ versus $8 \%$, and others: $18 \%$ versus $7 \%$.

Table 1 shows the prevalence of symptoms in the first $83 \mathrm{~A}$ and $\mathrm{E}$ patients (exposed) and the referent group with the relative rate for the exposed group. All except three of the 14 self reported symptoms were associated with attendance at the site. Headache, sore throat, shortness of breath, and weakness were highly significantly associated with attendance at the site $(\mathrm{p}<0.001)$.

The figure shows the frequency distribution of the CK concentrations. The median concentration was significantly higher in the early A and E patients (139 units) compared with the referent group (111 units; mean rank $\mathrm{p}=0.03)$. The difference was of the same magnitude (median CK 127 versus 148) when the analysis was confined to men. Three later $\mathrm{A}$ and $\mathrm{E}$ patients also had very high concentrations of CK (1188, 5841, and 6294). Among those with very high CK three were at the site of the incident, of whom two were thought to have had low level exposure and the other very low level exposure, and the fourth (who remained asymptomatic) was in a

Table 2 Symptom prevalence ( $n$ ) by exposure category among all who attended the site

\begin{tabular}{lccccc}
\hline \multicolumn{5}{c}{ Exposure category symptom prevalence $(n(\%))$} & \\
\cline { 2 - 5 } Symptom & $\begin{array}{l}\text { High } \\
(n=50)\end{array}$ & $\begin{array}{l}\text { Medium } \\
(n=96)\end{array}$ & $\begin{array}{l}\text { Low } \\
(n=47)\end{array}$ & $\begin{array}{l}\text { Very low } \\
(n=31)\end{array}$ & $\begin{array}{l}\text { p Value } \\
\text { (for trend) }\end{array}$ \\
\hline Abdominal pain & $5(10.0)$ & $9(9.4)$ & $3(6.4)$ & $3(9.7)$ & 0.75 \\
Chest pain & $11(22.0)$ & $15(15.6)$ & $3(6.4)$ & $2(6.5)$ & 0.01 \\
Cough & $14(28.0)$ & $15(15.6)$ & $7(14.9)$ & $5(16.1)$ & 0.16 \\
Diarrhoea & $4(8.0)$ & $13(13.5)$ & $5(13.6)$ & $2(6.5)$ & 0.77 \\
Drowsiness & $12(24.0)$ & $17(17.7)$ & $6(12.8)$ & $4(12.9)$ & 0.13 \\
Headache & $23(46.0)$ & $41(42.7)$ & $15(31.9)$ & $10(32.3)$ & 0.10 \\
Itchy eyes & $9(18.0)$ & $17(17.7)$ & $7(14.9)$ & $3(9.7)$ & 0.30 \\
Nausea & $6(12.0)$ & $9(9.4)$ & $3(6.4)$ & $2(6.5)$ & 0.30 \\
Palpitations & $3(6.0)$ & $5(5.2)$ & $2(4.3)$ & $1(3.2)$ & 0.54 \\
Shortness of breath & $16(32.0)$ & $15(15.6)$ & $7(14.9)$ & $2(6.5)$ & 0.005 \\
Skin rash & $4(8.0)$ & $10(10.4)$ & $7(14.9)$ & $1(3.2)$ & 0.85 \\
Sore throat & $24(48.0)$ & $30(31.3)$ & $11(23.4)$ & $3(9.7)$ & 0.0002 \\
Vomiting & $3(6.0)$ & $4(4.2)$ & $1(2.1)$ & $1(3.2)$ & 0.40 \\
Weakness & $13(26.0)$ & $18(18.8)$ & $7(14.9)$ & $5(16.1)$ & 0.20 \\
Wheeze & $8(16.0)$ & $12(12.5)$ & $6(12.8)$ & $1(3.2)$ & 0.13 \\
\hline
\end{tabular}

room adjacent to the mortuary not wearing protective clothing. The postmortem examinations were carried out on the two dead workers by pathologists wearing protective clothing.

Among all people who were at the site of the incident, 50 were classified as having a high exposure, 96 medium exposure, 46 low exposure, and 31 very low exposure. Twenty eight people were potentially exposed off site, either through taking waste from the site by tanker or in handling the bodies at the hospital, and exposure data were missing for three people. The most common symptoms reported by workers who attended the incident were headache $(\mathrm{n}=104,41 \%)$, followed by sore throat $(n=78,31 \%)$, cough $(n=47,19 \%)$, shortness of breath $(n=46,18 \%)$, and weakness $(n=46$, $18 \%$ ). Table 2 shows the symptom prevalence for those for whom it was possible to ascribe an exposure category and the $\mathrm{p}$ value from $\chi^{2}$ tests for trend. Table 3 shows the relation between grouped distance from the chamber and prevalence of symptoms, and the $\mathrm{p}$ value from $\chi^{2}$ tests for trend. Both tables show a significant association between shortness of breath or sore throat and estimates of exposure, but no association between severity of exposure and the reporting of headache. There was no difference between the mean $\mathrm{CK}$ values for the exposure categories and no correlation between concentrations of $\mathrm{CK}$ and reported headache, shortness of breath, or sore throat.

Results from GC-MS were obtained from 225 blood samples in the exposed group. Freon-11 was detected in two blood samples at concentrations of $2 \mu \mathrm{M}$. $\alpha$-Methyl styrene was not detected in any blood sample. Styrene was not detected in any blood sample at a detection concentration of $1 \mu \mathrm{M}$ and none of the 40 urine samples analysed for atrolactic acid had concentrations above the level of detection (20 $\mu \mathrm{M})$. Analyses for myoglobin and fluoride concentrations in 269 urine samples were within or very close to the normal range. 
Table 3 Prevalence of symptoms by grouped distance from chamber in all who attended the site

\begin{tabular}{lcccccc}
\hline \multicolumn{7}{c}{ Prevalence by grouped distance $(n(\%))$} \\
\cline { 2 - 5 } Symptom & $\begin{array}{l}0-4 m \\
(n=44)\end{array}$ & $\begin{array}{c}5-9 m \\
(n=35)\end{array}$ & $\begin{array}{l}10-19 m \\
(n=41)\end{array}$ & $\begin{array}{l}20-39 m \\
(n=60)\end{array}$ & $\begin{array}{l}40-300 m \\
(n=57)\end{array}$ & p Value \\
\hline Abdominal pain & $4(9.1)$ & $3(8.6)$ & $6(14.6)$ & $5(8.3)$ & $2(3.5)$ & 0.40 \\
Chest pain & $8(18.2)$ & $4(11.4)$ & $8(19.5)$ & $7(11.7)$ & $3(5.3)$ & 0.07 \\
Cough & $8(18.2)$ & $8(22.9)$ & $10(24.4)$ & $7(11.7)$ & $10(17.5)$ & 0.46 \\
Diarrhoea & $4(9.1)$ & $7(20.0)$ & $8(19.5)$ & $6(10.0)$ & $2(3.5)$ & 0.12 \\
Drowsiness & $8(18.2)$ & $6(17.1)$ & $8(19.5)$ & $12(20.0)$ & $8(14.0)$ & 0.72 \\
Headache & $19(43.2)$ & $14(40.0)$ & $24(58.5)$ & $19(31.7)$ & $18(31.6)$ & 0.11 \\
Itchy eyes & $8(18.2)$ & $8(22.9)$ & $13(31.7)$ & $5(8.3)$ & $5(8.8)$ & 0.03 \\
Nausea & $5(11.4)$ & $4(11.4)$ & $5(12.2)$ & $5(8.3)$ & $3(5.3)$ & 0.23 \\
Palpitations & $3(6.8)$ & $1(2.9)$ & $3(7.3)$ & $3(5.0)$ & $2(3.5)$ & 0.59 \\
Shortness of breath & $12(27.3)$ & $6(17.1)$ & $10(24.4)$ & $10(16.7)$ & $5(8.8)$ & 0.02 \\
Skin rash & $4(9.1)$ & $4(11.4)$ & $5(12.2)$ & $8(13.3)$ & $2(3.5)$ & 0.45 \\
Sore throat & $17(38.6)$ & $12(34.3)$ & $16(39.0)$ & $16(36.7)$ & $10(17.5)$ & 0.01 \\
Vomiting & $2(4.5)$ & $3(8.6)$ & $3(7.3)$ & $1(1.7)$ & $1(1.8)$ & 0.17 \\
Weakness & $9(20.5)$ & $6(17.1)$ & $12(29.3)$ & $10(16.7)$ & $8(14.0)$ & 0.38 \\
Wheeze & $6(13.6)$ & $6(17.1)$ & $3(7.3)$ & $10(16.7)$ & $5(8.8)$ & 0.54 \\
\hline
\end{tabular}

\section{Discussion}

Recognising and declaring serious chemical incidents may not always be easy and the police, the Health and Safety Executive, local authorities, health authority, and the water company all have responsibilities in the management of them. The United Kingdom National Health Service guidance on planning for major incidents requires health authorities to ensure that satisfactory arrangements are in place for handling the public healthcare aspects of response to chemical incidents ${ }^{2}$ and the role of the public health team in chemical incidents has been previously described..$^{3-5}$ In the initial phase of this incident, there was no agreement that hazardous chemicals were involved or that there was a risk to emergency personnel present at the site. Rapid environmental monitoring was undertaken, but there was uncertainty about the clinical importance of the amount of chemicals identified and whether there had been further chemical reactions if high temperatures had been generated by a faulty pump. Descriptive case studies of the early patients at the $\mathrm{A}$ and $\mathrm{E}$ department suggested the presence of an unknown chemical agent. This in turn alerted the emergency responders to alter the acute operational management of the incident, by increasing the distance of the police cordon from the chamber and advising that protective clothing with breathing apparatus was used within the cordon.

The analytical epidemiology showed that self reported symptoms, especially headache, sore throat, and shortness of breath were more common, and serum creatinine phosphokinase concentrations were significantly increased in emergency personnel who attended the site compared with emergency personnel who did not. The prevalence of the symptoms shortness of breath and sore throat showed a decreasing trend with decreasing exposure to the chamber whether estimated by direct distance or predefined exposure category.

At the coroner's inquest, held in April 1998, the inquest was told that more than three tonnes of Freon-11 had gone missing from a nearby chemical company where there had been a Freon spill months earlier. The cause of death of the two men was given as poisoning by Freon-11, and the inquest returned a verdict of unlawful killing. Freon is a fluorinated hydrocarbon (trichloromonofluoromethane) used as a refrigerant and ingredient of some fire extinguishers and aerosols. It is a gas at ambient temperatures and inhalation of fluorocarbons at low concentrations is associated with eye, nose, and throat irritation, headache (reported at high frequency) palpitation, dizziness, and light headedness. Chest tightness, cough, shortness of breath, and nausea may also occur. ${ }^{6} 7$ High concentrations of fluorocarbons, particularly in poorly ventilated areas, are associated with ventricular arrythmias, pulmonary oedema, and sudden death. ${ }^{7-9}$ Another possibility is that Freon-11 degraded in the sewer to form highly irritant breakdown products. For example, the methanogenic archaebacterium Methanosarcina barkeri (which has been identified in sewers in the United Kingdom) catalyses the degradation of Freons to fluoride. ${ }^{10}$

The symptoms experienced by personnel attending the site were consistent with exposure to fluorocarbons, although in the absence of direct evidence of exposure from biological samples it is not possible to ascribe cause. However, the delay in taking samples after exposure, coupled with the short half life of fluorocarbons in the body means that the negative biological samples cannot be taken as evidence of non-exposure. Interestingly, symptoms were related to indirect measures of exposure (direct distance and predefined categories) although some of the relations are not very strong. There is a possibility of misclassification of exposure based on a single point of exposure. Tankering operations may have resulted in exposure and might explain the exposure of one person who was at the site, not near the chamber but who was in the direct line of release from a "gully sucker" and who developed severe respiratory symptoms and widespread ulceration discovered on bronchoscopy. Several months after the incident, sampling of the sludge in the sewer chamber still showed very high concentrations of Freon. It is thought that a malfunctioning sewer pump allowed Freon to back up in the Victorian drainage system in the area and there may have been other sites of exposure from cracked piping which might explain why three of the 25 nearby residents had minute traces of Freon (1-2 $\mu \mathrm{M}) 9$ days after the initial incident, having not been within 100 metres of the sewer chamber at any time.

Medical surveillance was undertaken after the incident on personnel either complaining of symptoms or with abnormal blood tests. Creatine phosphokinase and urinary myoglobin were measured because of isolated reports of rhabdomyolysis after exposure to fluorocarbons. ${ }^{911}$ Other chemicals known to cause rhabdomyolysis were not identified in the sewer. The high concentrations of $\mathrm{CK}$ found in the early patients at $\mathrm{A}$ and $\mathrm{E}$ and the very high concentrations in the three later patients may 
indicate that subclinical muscle damage might occur after exposure to fluorocarbons. However, the results must be interpreted with caution in view of the possibility of other causes of increased CK-for example, strenuous exertion. The serum from the non-exposed emergency personnel redefined normal hospital reference values for a young physically active population. Concentrations of $\mathrm{CK}$ have been shown to be increased in athletes ${ }^{12}$ and many of the emergency personnel were found to be keen sportsmen as well as being physically active during working hours. Unnecessary follow up of healthy, emergency personnel with explained increased concentrations of CK was, therefore, avoided.

Publication of field epidemiological studies have an accepted role in improving investigation and control of infectious disease. By contrast, there are far fewer reported investigations of chemical incidents, perhaps because of difficulties in defining exposure or because measuring health outcomes in a community may require additional resources. Toxicological data on many chemicals is often scant, based on healthy volunteers or occupational experience. Investigation and reporting of community exposures improve scientific knowledge $\mathrm{e}^{13} 14$ and help others in the preparation for an emergency response.

Preparation for epidemiological investigation should be included in the planning phase for serious chemical incidents and its importance understood by all emergency services. The descriptive and analytical study undertaken during this incident immediately effected the operational management of the incident and clinical management of people presenting at the $\mathrm{A}$ and $\mathrm{E}$ department.

\section{Key messages}

(1) Descriptive epidemiology helps management decisions in acute chemical incidents.

(2) Normal biochemical values derived from referent groups may be needed to identify true morbidity.

(3) More reports of the management of, and outcomes from, chemical incidents are needed in peer reviewed journals to continually improve best practice.

We thank Dr Annie Delahunty, Dr Steven Monaghan, and Professor Palmer for advice on study design and implementation.

1 Watt MM, Watt S, Seaton A. Episode of toxic gas exposure in sewer workers. Occup Environ Med 1997;54:277-80.

2 World Health Organisation. Biological monitoring of chemcial exposure in the workplace. Vol 1. Geneva: WHO, 1996.

3 NHS Management Executive. Health service guidelines. Arrangements to deal with health aspects of chemical contamination incidents - HSG(93) 38. Heywood: NHSME, 1993.

4 Gunnell DJ. The public health physician's roles in chemical incidents. F Public Health Med 1993;15:352-7.

5 Baxter PJ, Heap BJ, Rowland MGM, et al. Thetford plastics fire, October 1991: the role of the preventative medical team in chemical incidents. Occup Environ Med 1995.52:694-8.

6 Holness DL, House RA. Health effects of Halon 1301 exposure. F Occup Med 1992;34:722-5.

7 Lerman Y, Winkler E, Tirosh MS, et al. Fatal accidental inhalation of bromochloro difluoromethane (Halon 1211). Hum Exp Toxicol 1991;10:125-8.

8 Thompson JF, Snodgrass WR. Inflatable toys: an unusual source of freon toxicity [abstract]. Vet Hum Toxicol 1991;33: 363.

9 Brady WJ, Stremski E, Eljiek L, et al. Freon inhalation abuse presenting with ventriacular fibrillation. Am $\mathcal{F}$ Emerg Med 1994;12:533-6.

10 Krone UE, Thauer RK. Dehalogenation of trichlorofluoromethane (CFC-11) by Methanosarcina barkeri. FEMS Microbiol Lett 1992;69:201-4.

11 Denborough MA, Hopkinson KC. Firefighting and malignant hyperthermia. BMF 1988;296:1442-3.

12 Siegel AJ, Sholar M, Yang J, et al. Elevated serum cardiac markers in asymptomatic marathon runners after competition: is the myocardium stunned? Cardiology 1997; 88:487-91.

13 Hoque A, Sigurdson AJ, Burau KD, et al. Cancer among a Michigan cohort exposed to polybrominated biphenyls in

14 Jarvis SN, Straube RC, Williams ALJ, et al. Illness associated with contamination of drinking water supplies with phenol. BMF 1985;290:1800-2.

\section{6th European Forum on Quality Improvement in Health Care}

\section{Thursday 29 - Saturday 31 March 2001. Bologna, Italy Call for papers}

For full information contact: BMA/BMJ Conference Unit, BMA House, Tavistock Square, London, WC1H 9JP, UK. Telephone +44 (0) 207383 6409; fax: +44 (0) 207383 6869; email quality@bma.org.uk Or go to the website for full details: www.quality.bmjpg.com 